\title{
Multiple Antenna Time Reversal Transmission In Ultra-Wideband Communications
}

\author{
Yuanwei Jin, Yi Jiang, and José M. F. Moura \\ Electrical \& Computer Engineering \\ Carnegie Mellon University \\ Pittsburgh, PA 15213
}

\begin{abstract}
In this paper we study the multiple antenna time reversal downlink transmission in an ultra-wideband (UWB) communication system which consists of access points and users. The access point has multiple antennas and the user has a single antenna. We design the UWB beamformer that focuses on the intended user while minimizing its interference on unintended users and eavesdropping access points. We show that the designed UWB beamformer is equivalent to the time reversal focusing and nulling schemes and yields better performance than the conventional delay line wideband beamformer. We verify our results using experimentally measured electromagnetic data in an indoor environment.
\end{abstract}

\section{INTRODUCTION}

Ultrawideband (UWB) systems are based on the transmission of extremely low-power short-pulses that occupy a very large bandwidth. They are being considered for the next generation of high data rate wireless short range communication systems. With an ultra-wide bandwidth signal, the dense multipath components can be resolved. Thus, a RAKE receiver can be used for signal demodulation. However, a major design challenge of a UWB system is the large delay spread of the channel and a large number of resolvable paths in a typical indoor environment. As a result, the channel estimation and equalization may require a great amount of numerical calculation power.

Time reversal techniques have been applied in acoustics [1] and radar applications [2]. In time-reversal, a signal is precoded such that it focuses both in time and space. The application of time reversal with MMSE equalization to UWB communications is discussed in [3]. A multiuser scenario using time reversal in UWB is reported in [4]. Experimental studies of time reversal in ultrawideband communications are reported in [5]. These papers discuss the benefit of time reversal temporal focusing or spatial focusing that can shorten the channel response. As a result, the receiver complexity is reduced.

Multiple antennas have been used in wireless links of communications systems to suppress or cancel interference and to achieve improved performance by diversity or beamforming. MIMO techniques can be applied to UWB systems to extend the system RF range through spatial diversity, to increase throughput that is limited by the channel excess delay through spatial multiplexing [6]. This paper focuses on wideband transmit beamforming among all alternative MIMO techniques including space time coding or spatial multiplexing.

In this paper, we consider a UWB communication system in downlink, i.e., from an access point (AP) to users. Each access point has multiple antennas, while each user has a single antenna. The contributions of this paper are as follows. We design UWB beamformers to increase the intended user signal power without adding power to the unintended users and adjacent access points. We show that the proposed beamforming is equivalent to the time reversal focusing and nulling schemes. Time reversal focusing improves the channel link while time reversal nulling reduces interference among user streams. We validate our algorithms by measuring a UWB indoor wireless channel in the electromagnetic domain in our laboratory. Experimental measurement and numerical simulation demonstrate the effectiveness of the proposed broadband beamforming scheme in a typical dense multipath environment for a UWB communication system.

\section{Time Reversal Focusing AND Nulling}

It is well know that in optics, time reversal is equivalent to phase conjugation in the frequency domain. However, this does not reveal the whole picture of time reversal. From a signal processing perspective, time reversal is an optimal waveform transmission scheme. By probing the scattering channel iteratively, the time-reversed signal best matches with the target channel response.

Time reversal focusing refers to temporal or spatial energy concentration. In a multipath rich environment, the channel appears to be time dispersive. Represented as an FIR filter, the channel has a long delay spread. A convolution with a timereversed channel response is equivalent to auto-correlation. The auto-correlation of the channel manifests itself with short time support and short spatial support. The focusing becomes effective for a multipath rich channel, particularly in ultrawideband communications.

In contrast, nulling refers to anti-focusing. This strategy is useful for avoiding additional power on unintended users, which is equivalent to increasing the capacity of a communication system.

\section{System ModeL}

We consider the situation shown in Fig. 1 where an Access Point (AP) communicates with two users. The AP has $N$ antennas; each user has one antenna. We consider the case where the AP applies time reversal coefficients and focuses on user $m$. Each of the $N$ streams of symbols is then used to modulate a short-duration UWB pulse. 


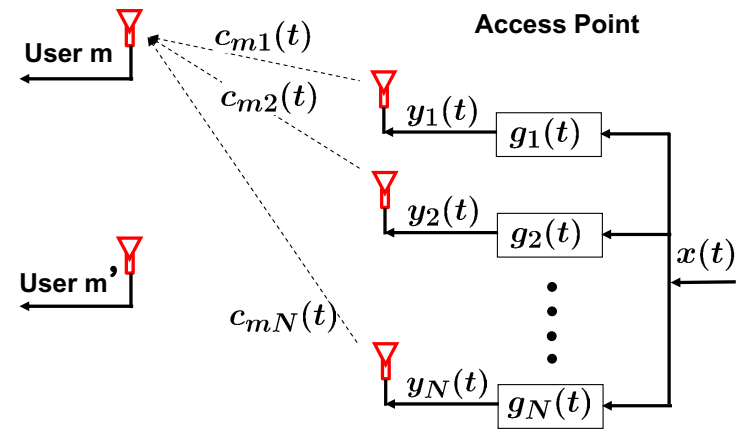

Fig. 1. A wideband beamformer. Filters $g_{n}(t)$ are designed to focus energy on user $m$.

\section{A. Modulated signal}

The pulse amplitude modulated (PAM) signal from the transmit antenna is expressed as

$$
x(t)=\sum_{k=-\infty}^{k=\infty} \sum_{m} \sqrt{E_{s}} s^{(m)}(k) p^{(m)}\left(t-k T_{\mathrm{PRI}}\right),
$$

where $s^{(m)}(k)$ is the $k$-th information symbol for user $m$, whose average energy of the signal constellation is normalized to unity. $E_{s}$ is the total energy of the basic pulse $p^{(m)}(t)$ for user $m$. The pulse has a very narrow non-zero time support $T_{p}$ relative to the pulse repetition interval $T_{\mathrm{PRI}}$, i.e., $T_{p}<T_{\mathrm{PRI}}$.

\section{B. UWB transmit beamforming and time reversal}

The generated signal will go through the wideband beamformer shown in Fig. 1, where a bank of filters $g_{n}(t)$ are applied to the signal $x(t)$ and transmitted by the corresponding antenna. We refer to this process as transmit wideband beamforming. In general, a beamformer can be implemented at a transmitter or a receiver. Conventionally, in receive beamforming, each filter $g_{n}(t)$ can be substituted by a delay in order to compensate for the different signal travel times among the antenna elements and to coherently summing across all branches. This is called the delay-sum beamformer [7]. However, interferers can not be suppressed only by delays. In general, a filter must be used. In transmit beamforming, we adopt the same idea by using filters $g_{n}(t)$. However, the filter $g_{n}(t)$ must adapt to the wireless channels. Next, we discuss how to design optimal filter $g_{n}(t)$ in the case of a single user and multiple users. For convenience, we will rewrite the signal model in the frequency domain by the Fourier transform. Hence, Eqn. (1) becomes

$$
X(\omega)=\sum_{k=-\infty}^{k=\infty} \sum_{m} \sqrt{E_{s}} s^{(m)}(k) P^{(m)}(\omega) e^{-j \omega k T_{\mathrm{PRI}}} .
$$

1) Single User: Let $G_{n}(\omega)$ and $C_{m, n}(\omega)$ be the frequency representation of the filter $g_{n}(t)$ and the channel $c_{m, n}(t)$ between the $n$-th antenna to the $m$ th user, we have the following expression

$$
Y_{n}(\omega)=X(\omega) G_{n}(\omega),
$$

and the total received signal at user $m$, excluding noise, is

$$
R_{m}(\omega)=\sum_{n=1}^{N} G_{n}(\omega) C_{m, n}(\omega) X(\omega) .
$$

At the user receiver, we apply the matched filter $P^{(m) *}(\omega)$. Thus, we obtain the demodulated signal as follows:

$$
\begin{aligned}
R_{m}(\omega)= & \sum_{k} \sqrt{E_{s}} s^{(m)}(k) e^{-j \omega k T_{\mathrm{PRI}}} \\
& \times\left|P^{(m)}(\omega)\right|^{2} \sum_{n=1}^{N} C_{m, n}(\omega) G_{n}(\omega) .
\end{aligned}
$$

For simplicity, we assume that the modulating pulse $p^{(m)}(t)$ has a brick wall spectrum, i.e., $P^{(m)}(\omega)=1$. Thus,

$$
R(\omega)=\eta_{m}(\omega) \sum_{k} \sqrt{E_{s}} s(k) e^{-j \omega k T_{\mathrm{PRI}}},
$$

where the overall channel coefficient for user $m$ is given by

$$
\eta_{m}(\omega)=\sum_{n=1}^{N} C_{m, n}(\omega) G_{n}(\omega)
$$

The filter $G_{n}(\omega)$ is designed by

$$
G_{n}(\omega)=\arg \max _{G_{n}(\omega)} \int\left|\sum_{n=1}^{N} C_{m, n}(\omega) G_{n}(\omega)\right|^{2} d \omega,
$$

with the unity filter power constraint

$$
\int\left|G_{n}(\omega)\right|^{2} d \omega=1
$$

Employing the Lagrange rule and the theory of functional derivative of complex variables [8], we can show that the optimal filter $G_{n}(\omega)$ is

$$
G_{n}(\omega)=\gamma_{n} C_{m, n}^{*}(\omega),
$$

where $\gamma_{n}$ is a constant which ensures that the amount of transmit power from each antenna remains the same. Noticing that the phase conjugation term $C_{m, n}^{*}(\omega)$ in Eqn. (10) is equivalent to time reversal, we conclude that the optimal filter $g_{n}(t)$ in the time domain is a time-reversed version of the channel response $c_{m, n}(t)$, i.e.,

$$
g_{n}(t)=\gamma_{n} c_{m, n}(-t) \text {. }
$$

2) Multiple users and APs: Each AP has a fixed coverage area. Fig. 2 depicts a scenario that AP1 communicates with user $m$. The channel response from AP1 to user $m$, from AP1 to user $m^{\prime}$ is denoted by $\mathbf{c}_{m}(\omega)$ and $\mathbf{c}_{m^{\prime}}(\omega)$, respectively, where

$$
\begin{aligned}
\mathbf{c}_{m}(\omega) & =\left[C_{m, 1}(\omega), C_{m, 2}(\omega), \cdots, C_{m, N}(\omega)\right]^{T} \\
\mathbf{c}_{m^{\prime}}(\omega) & =\left[C_{m^{\prime}, 1}(\omega), C_{m^{\prime}, 2}(\omega), \cdots, C_{m^{\prime}, N}(\omega)\right]^{T}
\end{aligned}
$$

In a multiuser scenario, the AP can schedule its transmission to the users with good channel conditions to improve the system throughput. This form of selection diversity is called multiuser diversity [9]. Appropriate beamforming schemes can be chosen to avoid interference among user streams. Fig. 2 


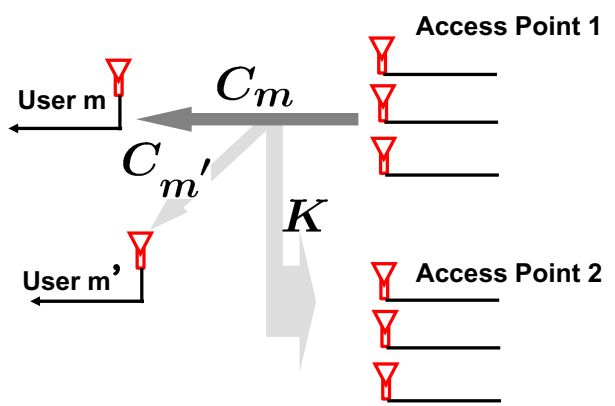

Fig. 2. Capacity improvement using time reversal focusing and nulling. AP1 communicates with user $m$. AP1 focuses its energy on user $m$ while minimizing its impact on user $m^{\prime}$ and a nearby AP2. The channel responses between AP1, user $m$, user $m^{\prime}$ and AP2 are $\mathbf{C}_{m}(\omega), \mathbf{C}_{m^{\prime}}(\omega)$, and $\mathbf{K}(\omega)$ at frequency $\omega$, respectively.

depicts a scenario that AP1 avoids the unintended user $m^{\prime}$ while focusing on the intended user $m$ through beamforming schemes.

We will also consider how the beamforming scheme affects the channel from AP1 to an eavesdropping AP2. We assume that AP2 has $N$ antennas. Then the channel between two APs is denoted by a $N$ by $N$ matrix $\mathbf{K}(\omega)$ whose $(i, j)$ th entry is the channel frequency response between $j$-th antenna of AP1 and $i$-th antenna of AP2. Ideally, AP1 should be able to improve the channel to user $m$ without adding additional power to user $m^{\prime}$ and AP2. This implies that the UWB beamformer focuses its energy on the intended user $m$ while minimizing or nulling its energy on the unintended user $m^{\prime}$ and an eavesdropping AP2.

In a multiple access network, adjacent APs may share the same channel. If one AP detects packets sent by another AP, a mechanism of controlling access is invoked. For example, carrier sense multiple access (CSMA) in IEEE 802.11 proposes transmission deferral. This deferral reduces the capacity available to each AP. Usually, system design will use different frequency channels. However, this strategy is not appropriate in ultra-wide band systems. To overcome this problem, we would use time reversal adaptive interference cancellation (TRAIC) arrays to prevent APs to detect one another's packets, thereby increasing the system capacity.

Next, we will design the beamformer for AP1 to achieve the goal of focusing on the intended user $m$ while suppressing the energy delivered to user $m^{\prime}$ and an eavesdropping AP2. Let

$$
\mathbf{g}(\omega)=\left[G_{1}(\omega), \cdots, G_{N}(\omega)\right]^{T}
$$

be the collection of filter frequency response. Similar to Eqn. (7), the transmitted signal arriving at AP2 and user $m$ is given by

$$
\begin{aligned}
\mathbf{i}(\omega) & =\mathbf{K}(\omega) \mathbf{g}(\omega)+\mathbf{C}_{m^{\prime}}(\omega) \mathbf{g}(\omega) \\
& =\mathbf{R}(\omega) \mathbf{g}(\omega),
\end{aligned}
$$

where

$$
\begin{aligned}
\mathbf{C}_{m^{\prime}}(\omega) & =\operatorname{diag}\left[C_{m^{\prime}, 1}(\omega), \cdots, C_{m^{\prime}, N}(\omega)\right] \\
\mathbf{R}(\omega) & =\mathbf{K}(\omega)+\mathbf{C}_{m^{\prime}}(\omega) .
\end{aligned}
$$

The filter design problem can be formulated by achieving two goals: focusing on the designated user and minimizing the unwanted interference to other users and the adjacent APs. Mathematically, it is equivalent to minimizing the total interfering signal power

$$
\mathbf{g}(\omega)=\arg \min _{\mathbf{g}(\omega)} \int\|\mathbf{i}(\omega)\|^{2} d \omega
$$

subject to the following constraint

$$
\int \mathbf{g}^{T}(\omega) \mathbf{c}_{m, n}(\omega) d \omega=\gamma \int\left\|\mathbf{c}_{m}(\omega)\right\|^{2} d \omega .
$$

$\gamma$ is the normalization factor similar to $\gamma_{n}$ in (10). Employing the Lagrange rule and the theory of functional derivative of complex variables [8], we can solve for $\mathbf{g}(\omega)$ :

$$
\mathbf{g}(\omega)=\frac{\gamma \int\left\|\mathbf{c}_{m}(\omega)\right\|^{2} d \omega\left[\left(\mathbf{R}^{H}(\omega) \mathbf{R}(\omega)\right)^{-1} \mathbf{c}_{m}^{*}(\omega)\right]}{\int \mathbf{c}_{m}^{T}(\omega)\left(\mathbf{R}^{H}(\omega) \mathbf{R}(\omega)\right)^{-1} \mathbf{c}_{m}^{*}(\omega) d \omega} .
$$

The phase conjugate appearing in Eqn. (21) indicates that the resulting filter is a time-reversal filter of $c_{m, n}(\omega)$. However, it is also clear that the signal power of AP1 leaking to AP2 and user $m^{\prime}$ is minimized. The time domain filter $g_{n}(t)$ can be designed by converting the frequency response of (21) into the time domain through the inverse Fourier transform.

\section{Symbol Detection}

The superfine time resolution in a pulsed UWB system typically results in a much greater number of resolvable paths than in traditional narrowband systems. Despite the significant differences between UWB and narrowband behaviors, existing work has shown that a tapped-delay-line model is appropriate to describe the UWB channels [10], [11]. The channel response can be written as

$$
c_{m, n}(t)=\sum_{l=0}^{L-1} c_{m, n}(l) \delta\left(t-l \tau_{p}\right),
$$

where $c_{m, n}(l)$ represents the channel coefficients of the $l$ th path for the signal from the $n$-th antenna to the $m$-th user. In a multipath rich environment, the number of resolvable paths $L$ could be very large. Practical values of $L$ depend on the root-mean-squared delay spread of the channel, multipath resolution, and the range of attenuation from the statistically strongest path to the weakest path. Early experimental measurement indicated that the average number of resolvable path is about $L=40$ with a $10 \mathrm{~dB}$ range (see, e.g., [11]). A RAKE receiver is commonly used to collect these paths coherently to increase the signal strength. However, the receiver complexity is limited by the battery power, particulary in a user terminal.

In this section, we focus on the symbol detection problem in downlink through UWB transmit beamforming. The filter response $g_{n}(t)$ is constructed by taking the inverse Fourier transform of (10) for a single user case or of (21) for a multiple user case. We further assume that the channel between the users and the APs are stationary over a period of a block of symbols. Thus, the UWB channel can be estimated semiblindly [12] or by pilot waveforms [13]. 
Single user The $n$th filter $g_{n}(t)$ computed by (10) is the time-reversed channel response, written as

$$
g_{n}(t)=c_{m, n}\left(T_{\mathrm{PRI}}-t\right)=\sum_{l=0}^{L-1} c_{m, n}(l) \delta\left(T_{\mathrm{PRI}}-t-l \tau_{p}\right)
$$

where we choose $T_{\mathrm{PRI}} \geq(L-1) \tau_{p}$ the maximum delay. This implies that the combined channel and filter response has a shortened spread

$$
\begin{aligned}
f_{m, n}(t)= & \sum_{l=0}^{L-1} \sum_{l^{\prime}=0}^{L-1} c_{m, n}(l) c_{m, n}\left(l^{\prime}\right) \delta\left(t-l \tau_{p}\right) \\
& \delta\left(T_{\mathrm{PRI}}-t-l^{\prime} \tau_{p}\right) \\
= & \sum_{l=0}^{L-1} c_{m, n}^{2}(l) \delta\left(T_{\mathrm{PRI}}-t\right)+\sum_{l} \sum_{l^{\prime}} c_{m, n}(l) \\
& c_{m, n}\left(l^{\prime}\right) \delta\left(T_{\mathrm{PRI}}-t+l \tau_{p}-l^{\prime} \tau_{p}\right) .
\end{aligned}
$$

$f_{m, n}(t)$ is the autocorrelation of the channel $c_{m, n}(t)$ with its energy concentrating in a narrow time support. Let $L_{\text {tr }} \ll$ $L$ be the number of dominant component of $f_{m, n}(t)$, which can be defined, for example, as the number of components with $f_{m, n}(t)$ exceeding a threshold. In fact, we may select the single tap with the largest amplitude, i.e., $L_{\text {tr }}=1$, and

$$
\left.f_{m, n}(t)\right|_{t=T_{\mathrm{PRI}}}=\sum_{l=0}^{L-1} c_{m, n}^{2}(l) .
$$

This implies that the combined response $f_{m, n}(t)$ is significantly shortened relative to the channel response $c_{m, n}(t)$. Thus, the equalizer at the user receiver becomes very simple.

For convenience, we will replace $T_{\mathrm{PRI}}-t$ by $t$. Thus, we can re-write Eqn. (25) as follows,

$$
f_{m, n}(t) \approx \sum_{l=0}^{L_{\mathrm{tr}}-1} f_{m, n}(l) \delta\left(t-l \tau_{p}\right),
$$

where $f_{m, n}(l)$ is the coefficient of the $l$-th dominant path. The received signal at the designated user becomes

$$
r(t)=\sum_{n=1}^{N} \sum_{l=0}^{L_{\mathrm{tr}}-1} f_{m, n}(l) x\left(t-l \tau_{p}\right)+w(t) .
$$

The receiver consists of a matched filter that matches to the UWB pulse $p(t)$ and then sampled at each path interval. A simplified RAKE receiver that collects coherently $L_{\text {tr }}$ path signals is used. The information carrying symbols go through a decision device for a symbol-by-symbol detection.

Multiuser or APs In this case, the $n$th filter $g_{n}(t)$ computed by (21) is the channel response with time-reversal focusing (on the designated user $m$ ) and time-reversal nulling (of the other users and APs). We can write

$$
f_{m, n}(t)=g_{n}(t) \star c_{m, n}(t) .
$$

By choosing the proper channel length $\bar{L}$, we have the following approximation

$$
f_{m, n}(t) \approx \sum_{l=0}^{\bar{L}-1} f_{m, n}(l) \delta\left(t-l \tau_{p}\right) .
$$

Because the designed beamformer (21) achieves focusing, the receiver at user $m$ can again use the single tap equalizer, i.e., $\bar{L}=1$.

\section{A. Single User Detection and Error Analysis}

Next, we describe the mathematical model of the receiver. Due to space limitation, we only discuss the single user detection. The multi-user detection problem will be discussed elsewhere. The matched filter output at $l$ th path $r(l), l=$ $0, \cdots, L_{\text {tr }}-1$, can be written as

$$
r^{(m)}(l)=\sqrt{E_{s}} \sum_{n=1}^{N} f_{m, n}(l) s^{(m)}(k)+w(l),
$$

where $w(l)$ is a zero-mean white Gaussian noise $w(l) \sim$ $\mathcal{N}\left(0, N_{0}\right)$. Employing the likelihood ratio test principle, the estimated symbol becomes

$$
\widehat{s^{(m)}(k)}=\frac{\sum_{l=0}^{L \mathrm{tr}-1} r^{(m)}(l)}{\sqrt{E_{s}} \sum_{n=1}^{N} \sum_{l=0}^{L_{\mathrm{tr}}-1} f_{m, n}(l)} .
$$

Here we conduct the error analysis on the binary PAM scheme. The analysis can be extended to a general PAM scheme. Here the average symbol energy $E_{s}$ is replaced by the bit energy $E_{b}$, the instantaneous SNR is given by

$$
\gamma=\frac{E_{b}}{N_{0}}\left(\frac{1}{L \mathrm{tr}} \sum_{l=0}^{L \mathrm{tr}-1} \sum_{n=1}^{N} f_{m, n}(l)\right)^{2} .
$$

The bit error rate (BER) given a fixed set of channel coefficients is [14]

$$
P_{b}=Q(\sqrt{\gamma})=\frac{1}{\sqrt{2 \pi}} \int_{\sqrt{\gamma}}^{\infty} e^{-z^{2} / 2} d z .
$$

\section{Indoor Channel Measurement}

Measurements are conducted with a network analyzer in a rich scattering laboratory environment. The measured frequency range is $4-6 \mathrm{GHz}$ with $100 \mathrm{MHz}$ frequency resolution. The frequency resolution is determined by the coherence bandwidth of the multipath channel. Thus the channel has a total of 201 frequency points and is equivalent to having 201 taps in the time domain. The effective delay resolution is 0.5 ns. The environment is quasi-static during the measurements and only the stationary user device scenarios are used for the results presented in this paper. The AP has 4 antennas with inter-element spacing of $3.5 \mathrm{~cm}=0.58 \lambda_{c}$, where $\lambda_{c}=6 \mathrm{~cm}$, and is synthesized by moving a single antenna along a 1 meter long position slider. We choose the data measured at different locations of the position slider as the user data and the AP data. Thus, we obtain the measurement between one AP and two users, and the measurement between the two APs as illustrated in Fig. 2. Fig. 3 depicts the combined channel response based on our measured channel with a single user (upper figure by Eqn.(25)) and multiple users (lower figure by Eqn.(29)). Both cases achieve focusing, which implies that for the designated user, a simple single-tap equalizer can be implemented. 

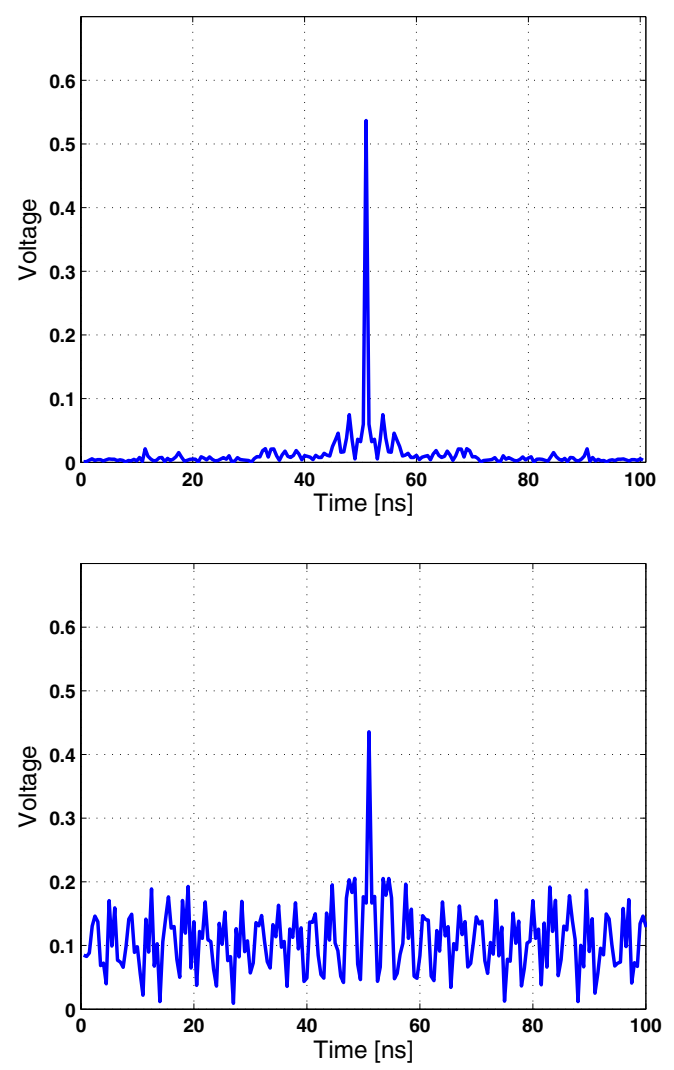

Fig. 3. Upper figure - The combined channel response of $f_{m, n}(t)$ achieves focusing (see 25). The channel shows a focusing spot. Lower figure - The combined channel response of $f_{m, n}(t)$ achieves focusing and nulling (see 29).

\section{NUMERICAL RESUlts}

We carry out numerical evaluation of the bit error rate analysis by inserting computer generated noise into the measured indoor channel response. We use binary PAM modulation. No channel coding is employed. Fig. 4 depicts the bit error rate of time reversal focusing (25), time reversal focusing and nulling (29), and a delay-line transmit wideband beamformer [15] for a single user detection. The delay-line transmit beamformer transmits signals with a constant transmission delay on all the antennas, i.e., the filter $g_{n}(t)$ is a simple delay. Thus, the user receiver must collect all the pulses in a dense multipath environment. Fig. 4 shows that, using a single-tap equalizer at the single user receiver, the time reversal focusing beamformer (Eqn. (10)) has a much lower bit error rate than the delay line beamformer. The nulling and focusing beamformer (Eqn. (21)) has a slight degradation than the focusing beamformer, but significantly outperforms the delay line beamformer.

\section{CONCLUSion AND Future WORK}

We derived transmit beamforming schemes for a single user and multiple user scenarios in UWB downlink transmission. We showed that the algorithms are equivalent to time reversal focusing and nulling and that they outperform the delay line wideband beamformer [15].

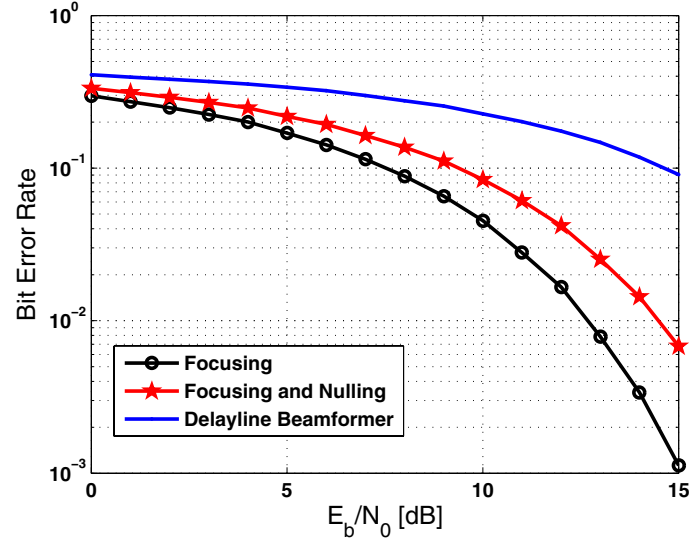

Fig. 4. Bit error rate plot for focusing beamformer (Eqn. (10)), focusing and nulling beamformer (Eqn. (21)), and delay-line beamformer.

This paper focused on the single user detection. However, time reversal focusing and nulling (see the focusing and nulling curve in Fig. 4 provides further benefit when multiple users are considered since the beamformer (Eqn. (21)) focuses on the designated user while minimizing its interference to other users, which improves the overall system capacity.

\section{REFERENCES}

[1] M. Fink, "Time reversed acoustics," Physics Today, vol. 50, no. 3, pp. 34-40, 1997.

[2] J. M. F. Moura and Y. Jin, "Time reversal detection: single antenna," IEEE Transactions on Signal Processing, vol. 54, no. 1, pp. 187-201, January 2007.

[3] T. Strohmer, M. Emami, J. Hansen, G. Papanicolaou, and A. J. Paulraj, "Application of time-reversal with MMSE equalizer to UWB communications," in IEEE Globecom. IEEE, 2004, pp. 3123-3127.

[4] H. T. Nguyen, I. Z. Kovacs, and P. C. F. Egers, "A time reversal transmission approach for multiuser UWB communications," IEEE Transactions on Antennas and Propagation, vol. 54, no. 11, pp. 32163223, November 2006.

[5] R. C. Qiu, C. Zhou, N. Guo, and J. Q. Zhang, "Time reveral with MISO for ultrawideband communications: experimental results," IEEE Antennas and Wireless Propagation Letters, vol. 5, no. 1, pp. 269-273, December 2006.

[6] L. Yang and G. B. Giannakis, "Ultra-wideband communications: an idea whose time has come," IEEE Signal Processing Magazine, pp. 26-54, November 2004.

[7] D. H. Johnson and D. E. Dudgeon, Array Signal Processing: Concepts and Techniques. Upper Saddle River, NJ: Prentice Hall, 1993.

[8] G. B. Arfken and H. J. Weber, Mathematical Methods for Physicists. Burlington, MA: Elesvier Academic Press, 2005.

[9] T. Yoo and A. Goldsmith, "Optimality of zero-forcing bemforming with multiuser diversity," in ICC 2005, vol. 1. IEEE, May 2005, pp. 542 546.

[10] C. Dajana, M. Z. Win, and A. F. Molisch, "The ultra-wideband indoor channel: from statistical model to simulations," IEEE Journal of Selected Areas Communications, vol. 20, no. 6, pp. 1247-1257, August 2002.

[11] A. F. Molisch, J. R. Foerster, and M. Pendergrass, "Channel models for ultra-wideband personal area network," IEEE Wireless Communications, vol. 10, no. 6, pp. 14-21, December 2003.

[12] J. Cai, X. Shen, J. W. Mark, H. Liu, and T. D. Todd, "Semiblind channel estimation for pulse-based ultra-wideband wireless communication system," IEEE Transactions on Vehicular Technology, vol. 55, no. 1, pp. 95-103, January 2006.

[13] L. Yang and G. B. Giannakis, "Optimal pilot waveform assisted modulation for ultrawideband communications," IEEE Transactions on Wireless Communications, vol. 3, no. 4, pp. 1236-1249, July 2004.

[14] J. G. Proakis, Digital Communications (3rd ed.). McGraw-Hill, 1995.

[15] M. Ghavami, L. B. Michael, and R. Kohno, Ultra Wideband Signals and Systems in Communication Engineering. West Sussex, UK: John Wiley \& Sons, 2004 